Communications in Physics, Vol. 24, No. 4 (2014), pp. 343-351

DOI:10.15625/0868-3166/24/4/5041

\title{
PHASE STRUCTURE OF BOSE - EINSTEIN CONDENSATE IN ULTRA - COLD BOSE GASES
}

TRAN HUU PHAT

Vietnam Atomic Energy Institute, 59 Ly Thuong Kiet, Hanoi, Vietnam

LE VIET HOA

Department of Physics, Hanoi University of Education, 136 Xuan Thuy, Cau Giay, Hanoi, Vietnam

DANG THI MINH HUE

Division of Physics, Hanoi Water Resources University, 175 Tay Son, Dong Da, Hanoi, Vietnam

E-mail: hue_dtm@wru.edu.vn

Received 02 October 2014

Accepted for publication 24 December 2014

\begin{abstract}
The Bose - Einstein condensation of ultra - cold Bose gases is studied by means of the Cornwall - Jackiw Tomboulis effective potential approach in the improved double - bubble approximation which preserves the Goldstone theorem. The phase structure of Bose - Einstein condensate associating with two different types of phase transition is systematically investigated. Its main feature is that the symmetry which was broken at zero temperature gets restored at higher temperature.
\end{abstract}

Keywords: Bose-Einstein condensation, superfluid and restoration phase transition.

\section{INTRODUCTION}

The experiments on Bose - Einstein condensation (BEC) of atomic vapors ${ }^{87} \mathrm{Rb}$ and ${ }^{23} \mathrm{Na}$ was successfully realized in 1995 [1,2]. Just after this first achievement a series of experiments [3], [6] dealing with BEC of systems composed of two distinct species of atoms revealed that the multi - component BEC is not a simple extension of the single component BEC. There arise many fascinating phenomena such as the quantum tunneling of spin domain [7], vortex configuration [8], phase segregation [9] and so on. This event marked a very rapid development in the study of quantum gases. The most attractive feature of all experiments realizing BEC in dilute ultra - cold gases is that almost every parameter of the system is controllable, thereby many novel phenomena, in particular, various types of phase transition, could be created experimentally by simply adjusting the model parameters. In parallel with experimental efforts we witness theoretical progresses [10, 21] aiming at describing different observed phenomena of multi - component systems as well as predicting new quantum effects. In this connection, to study the phase structure of Bose - Einstein condensates in ultra - cold gases, both experimental and theoretical, is of special interest. This 
paper is devoted to investigating the phase structure of one component Bose - Einstein condensate. To this end, let us begin with the dilute Bose gases given by the Lagrangian:

$$
L=\phi^{*}\left(-i \frac{\partial}{\partial t}-\frac{\nabla^{2}}{2 m}\right) \phi-\mu \phi^{*} \phi+\frac{\lambda}{2}\left(\phi^{*} \phi\right)^{2},
$$

where $\mu$ represents the chemical potential, $m$ represents the mass of Bose atom, and the coupling constant $\lambda$ is expressed through the $\mathrm{s}$ - wave scattering length a by

$$
\lambda=\frac{4 \pi \hbar^{2} a}{m} .
$$

For repulsive interaction $\lambda>0$.

The controllable parameters associating with the model (1) involve two model parameters $\mu, \lambda$ and the temperature $\mathrm{T}$.

This article is organized as follows. In section II based on [22,23] we establish the Cornwall - Jackiw - Tomboulis (CJT) effective potential for the system (1) in the improved double - bubble approximation together with the equations of state. The section III deals with the numerical study of various scenarios of phase transition. The conclusion is presented in section IV.

\section{EFFECTIVE POTENTIAL IN DOUBLE BUBBLE APPROXIMATION AND EQUATION OF STATE}

In the tree approximation the condensate density

$$
\rho_{0}=\phi_{0}^{2}
$$

corresponds to the local minimum of the potential. It fulfills

$$
\mu \phi_{0}-\frac{\lambda}{2} \phi_{0}^{3}=0
$$

yielding

for $\phi_{0} \neq 0$.

$$
\frac{\phi_{0}^{2}}{2}=\frac{\mu}{\lambda}
$$

Let us now focus on the calculation of the CJT effective potential in the double - bubble approximation [22]. At the first, the field operator is decomposed

$$
\phi=\frac{1}{\sqrt{2}}\left(\phi_{0}+\phi_{1}+i \phi_{2}\right)
$$

Inserting Eq. (6) into Eq. (1) we get, among other, the interaction Lagrangian

$$
L_{\text {int }}=\frac{\lambda}{2} \phi_{0} \phi_{1}\left(\phi_{1}^{2}+\phi_{2}^{2}\right)+\frac{\lambda}{8}\left(\phi_{1}^{2}+\phi_{2}^{2}\right)^{2},
$$

and the inverse propagator in the tree approximation in momentum space

$$
\begin{aligned}
D_{0}^{-1}(k) & =\left\|A_{i j}\right\|, \\
A_{11} & =\frac{\vec{k}^{2}}{2 m}-\mu+\frac{3 \lambda}{2} \phi_{0}^{2}, \\
A_{12} & =-A_{21}=-\omega,
\end{aligned}
$$




$$
A_{22}=\frac{\vec{k}^{2}}{2 m}-\mu+\frac{\lambda}{2} \phi_{0}^{2} .
$$

From Eq. (5) and Eq. (8) it follows that

$$
E=\sqrt{\frac{\vec{k}^{2}}{2 m}\left(\frac{\vec{k}^{2}}{2 m}+\lambda \phi_{0}^{2}\right)},
$$

which is the Bogoliubov dispersion relation for Bose condensate of ultra - cold Bose gases.

For small momenta Eq. (9) reduces to

$$
E \sim \sqrt{\frac{\lambda \phi_{0}^{2}}{2 m}} . k
$$

associating with the Goldstone boson due to the $\mathrm{U}(1)$ breaking.

Next the CJT effective potential is calculated in the double - bubble approximation in which the obtained self - energy is momentum independent. Therefore, we assume the ansatz for the propagator

$$
D^{-1}(k)=\left\|B_{i j}\right\|, B_{11}=\frac{\vec{k}^{2}}{2 m}+M_{1}, B_{22}=\frac{\vec{k}^{2}}{2 m}+M_{2}, B_{12}=-B_{21}=-\omega .
$$

Following closely [23] we arrive at the CJT effective potential $V_{\beta}\left(\phi_{0}, D\right)$ at finite temperature

$$
\begin{aligned}
V_{\beta}\left(\phi_{0}, D\right)= & -\frac{\mu}{2} \phi_{0}^{2}+\frac{\lambda}{8} \phi_{0}^{4}++\frac{1}{2} \int_{\beta} \operatorname{tr}\left[\ln D^{-1}+D_{0}^{-1} D-1\right] \\
& +\frac{3 \lambda}{8}\left[\int_{\beta} D_{11}(k)\right]^{2}+\frac{3 \lambda}{8}\left[\int_{\beta} D_{22}(k)\right]^{2} \\
& +\frac{\lambda}{4}\left[\int_{\beta} D_{11}(k)\right]\left[\int_{\beta} D_{22}(k)\right]
\end{aligned}
$$

where

$$
\int_{\beta} f(k)=T \sum_{n=-\infty}^{+\infty} \int \frac{d^{3} k}{(2 \pi)^{3}} f\left(\omega_{n}, \vec{k}\right) .
$$

Starting from Eq. (11) we get, respectively,

1. The gap equation

$$
\mu-\frac{\lambda}{2} \phi_{0}^{2}-\Sigma_{1}=0
$$

2. The Schwinger - Dyson equation

$$
D^{-1}(k)=D_{0}^{-1}(k)+\Sigma,
$$

in which

$$
\Sigma=\left\|\Sigma_{i j}\right\|, \Sigma_{11}=\Sigma_{1}, \Sigma_{22}=\Sigma_{2}, \Sigma_{12}=\Sigma_{21}=0,
$$

and

$$
\Sigma_{1}=\frac{3 \lambda}{2} \int_{\beta} D_{11}(k)+\frac{\lambda}{2} \int_{\beta} D_{22}(k),
$$




$$
\begin{aligned}
& \Sigma_{2}=\frac{\lambda}{2} \int_{\beta} D_{11}(k)+\frac{3 \lambda}{2} \int_{\beta} D_{22}(k), \\
& M_{1}=-\mu+\frac{3 \lambda}{2} \phi_{0}^{2}+\Sigma_{1}, M_{2}=-\mu+\frac{\lambda}{2} \phi_{0}^{2}+\Sigma_{2} .
\end{aligned}
$$

Then the explicit form of propagator comes out from combining Eqs. (13) and Eq. (14)

$$
\begin{aligned}
D^{-1} & =\left\|B_{i j}\right\|, B_{11}=\frac{\vec{k}^{2}}{2 m}-\mu+\frac{3 \lambda}{2} \phi_{0}^{2}+\Sigma_{1}, \\
B_{12} & =B_{21}=-\omega, B_{22}=\frac{\vec{k}^{2}}{2 m}-\mu+\frac{\lambda}{2} \phi_{0}^{2}+\Sigma_{2},
\end{aligned}
$$

which clearly indicates that the Goldstone theorem fails in this approximation. In order to restore it, we follow [24] to add an appropriate correction $\Delta V$ to $V_{\beta}\left(\phi_{0}, D\right)$, namely,

$$
\tilde{V}_{\beta}\left(\phi_{0}, D\right)=V_{\beta}\left(\phi_{0}, D\right)+\Delta V
$$

with

$$
\Delta V=-\frac{\lambda}{4}\left[P_{11}^{2}+P_{22}^{2}-2 P_{11} P_{22}\right], P_{a a}=\int_{\beta} D_{a a}(k) ; a=1,2 .
$$

It is easily checked that

$$
\begin{aligned}
\tilde{V}_{\beta}\left(\phi_{0}, D\right)= & -\frac{\mu}{2} \phi_{2}^{2}+\frac{\lambda}{8} \phi_{0}^{4}+\frac{1}{2} \int_{\beta} \operatorname{tr}\left[\ln D^{-1}+D_{0}^{-1}(k) D(k)-1\right] \\
& +\frac{\lambda}{8} P_{11}^{2}+\frac{\lambda}{8} P_{22}^{2}+\frac{3 \lambda}{4} P_{11} P_{22} .
\end{aligned}
$$

yielding

1. The gap equation

$$
-\mu+\frac{\lambda}{2} \phi_{0}^{2}+\Sigma_{2}^{*}=0
$$

or

$$
\frac{\phi_{0}^{2}}{2}=\frac{\bar{\mu}}{\lambda} ; \bar{\mu}=\mu-\Sigma_{2}^{*} .
$$

2. The SD equation

$$
D^{-1}(k)=D_{0}^{-1}(k)+\Sigma^{*},
$$

in which

$$
\begin{aligned}
\Sigma^{*} & =\left\|\Sigma_{i j}^{*}\right\|, \Sigma_{11}^{*}=\Sigma_{1}^{*}=\frac{\lambda}{2} P_{11}+\frac{3 \lambda}{2} P_{22}, \\
\Sigma_{12}^{*} & =\Sigma_{21}^{*}=0, \Sigma_{22}^{*}=\Sigma_{2}^{*}=\frac{3 \lambda}{2} P_{11}+\frac{\lambda}{2} P_{22} .
\end{aligned}
$$

Combining Eqs. (22), (23) and (24) provides

$$
\begin{aligned}
D^{-1}(k) & =\left\|B_{i j}\right\|, B_{11}=\frac{\vec{k}^{2}}{2 m}+M_{1}^{*}, \\
B_{22} & =\frac{\vec{k}^{2}}{2 m}, B_{12}=-B_{21}=-\omega, M_{1}^{*}=-\mu+\frac{3 \lambda}{2} \phi_{0}^{2}+\Sigma_{1}^{*} .
\end{aligned}
$$


It is immediately derived from Eq. (25) the dispersion relation

$$
E=\sqrt{\frac{\vec{k}^{2}}{2 m}\left(\frac{\vec{k}^{2}}{2 m}+M_{1}^{*}\right)}
$$

which preserves the Goldstone theorem

$$
E \sim \sqrt{\frac{M_{1}^{*}}{2 m}} \cdot k
$$

as $k \rightarrow 0$.

Due to Landau criteria for superfluidity the Bose condensate turns out to be superfluid in broken phase and its sound speed reads

$$
C=\sqrt{\frac{M_{1}^{*}}{2 m}}
$$

Ultimately the one - particle - irreducible effective potential $\tilde{V}_{\beta}\left(\phi_{0}\right)$ is read off from Eq. (20) with D fulfilling the SD equation Eq. (23),

$$
\begin{aligned}
\tilde{V}_{\beta}\left(\phi_{0}\right)= & -\frac{\mu}{2} \phi_{0}^{2}+\frac{\lambda}{8} \phi_{0}^{4}+\frac{1}{2} \int_{\beta} \operatorname{tr} \ln D^{-1}(k) \\
& +\frac{P_{11}}{2}\left(\frac{3 \lambda}{2} \phi_{0}^{2}-\mu-M_{1}^{*}\right)+\frac{P_{22}}{2}\left(\frac{\lambda}{2} \phi_{0}^{2}-\mu\right) \\
& +\frac{\lambda}{8}\left(P_{11}^{2}+P_{22}^{2}\right)+\frac{3 \lambda}{4} P_{11} P_{22} .
\end{aligned}
$$

Based on the effective potential $\tilde{V}_{\beta}\left(\phi_{0}, D\right)$ given in Eq. (20) various equations of state are easily derived as follows

- The pressure $\mathrm{P}$ is defined by

$$
P=-\tilde{V}_{\beta}\left(\phi_{0}, D\right)
$$

taken at minimum. Then the density of condensate reads

$$
\rho=-\frac{\partial \tilde{V}_{\beta}}{\partial \mu}=\frac{\phi_{0}^{2}}{2}+\frac{P_{11}+P_{22}}{2} .
$$

In term of density, the pressure (28) is expressed as

$$
P=\frac{\lambda}{2} \rho^{2}-\frac{1}{2} \int_{\beta} \operatorname{tr} \ln D^{-1}(k)+\lambda \rho P_{11}-\frac{\lambda}{2} P_{11}^{2} .
$$

The free energy $\mathrm{E}$ follows from the Legendre transform $E=\mu \rho-P$ leading to

$$
E=\frac{\lambda}{2} \rho^{2}+\frac{1}{2} \int_{\beta} t r D^{-1}(k)+\frac{\lambda}{2} P_{11}^{2} .
$$

Eqs.(30) and (31) are the basic equations of state of BEC which govern all phase transition processes of BEC. 
Starting from these equations let us consider respectively the high and low temperature behaviors of $\mathrm{P}$ and $\mathrm{E}$. To this end, we first mention that the symmetry $\mathrm{U}(1)$, broken at $T=0$, is restored at high temperature $T=T_{c}$ if $\phi_{0}^{2}=0$ as $T \rightarrow T_{c}$.

Using the high temperature expansion of all integrals appearing in Eq. (20) we arrive at

$$
T_{c}=2 \pi\left[\frac{\mu}{2 m^{3 / 2} \lambda \zeta(3 / 2)}\right]^{2 / 3}
$$

here $\zeta(z)$ is the zeta function of Riemann.

The behavior of pressure for high $\mathrm{T}$ just below the critical temperature is reduced

$$
P=\frac{\lambda}{2} \rho+\frac{m^{3 / 2} \zeta(5 / 2)}{2 \sqrt{2 \pi^{3}}} T^{5 / 2}+\frac{m^{3} \lambda[\zeta(3 / 2)]^{2}}{16 \pi^{3}} T^{3},
$$

which is the well -known result of Lee and Yang for Bose gas without invoking the double counting subtraction. The high $\mathrm{T}$ behavior of free energy is straightforwardly derived by means of the formula

$$
E=-\frac{\partial}{\partial \beta}[\beta P]_{\mu}
$$

which yields

$$
E=-\frac{\lambda}{2} \rho^{2}-\frac{3 m^{3 / 2} \lambda \rho \zeta(3 / 2)}{4 \sqrt{2 \pi^{3}}} T^{3 / 2}+\frac{3 m^{3 / 2} \zeta(5 / 2)}{4 \sqrt{2 \pi^{3}}} T^{5 / 2}+\frac{m^{3} \lambda[\zeta(3 / 2)]^{2}}{8 \pi^{3}} T^{3} .
$$

Next the behaviors of $\mathrm{P}$ and $\mathrm{E}$ are concerned for $T / \mu \leq 1$. At first we note that we have the approximate expression for $M_{1}^{*}$ in this regime

$$
M_{1}^{*} \simeq 2 \lambda \rho-\frac{2 \sqrt{2} M_{1}^{3 / 2} m^{3 / 2} \lambda}{3 \pi^{2}}-\frac{2 \sqrt{2} m^{3} \lambda \pi^{2}}{15 M_{1}^{* 5 / 2}},
$$

which requires a self - consistent solution for $M_{1}^{*}$ as function of density and temperature. The first approximation is chosen as $M_{1}^{*} \simeq 2 \lambda \rho$ leading to the low temperature behaviors of chemical potential, pressure and free energy:

- Chemical potential

$$
\mu \simeq \lambda \rho+\frac{4 m^{3 / 2} \lambda^{5 / 2} \rho^{3 / 2}}{3 \pi^{2}}+\frac{m^{3 / 2} \pi^{2}}{60 \lambda^{3 / 2} \rho^{5 / 2}} T^{4}
$$

- Pressure

$$
P=\frac{\lambda \rho^{2}}{2}+\frac{4 m^{3 / 2} \lambda^{5 / 2} \rho^{5 / 2}}{5 \pi^{2}}+\frac{m^{3 / 2} \pi^{2}}{36 \lambda^{3 / 2} \rho^{3 / 2}} T^{4}
$$

- Free energy

$$
E=\mu \rho-P=\frac{\lambda \rho^{2}}{2}+\frac{8 m^{3 / 2} \lambda^{5 / 2} \rho^{5 / 2}}{15 \pi^{2}}+\left(\frac{m^{3}}{45 \rho}-\frac{m^{3 / 2} \pi^{2}}{90 \lambda^{3 / 2} \rho^{3 / 2}}\right) T^{4} .
$$




\section{NUMERICAL STUDY}

Starting from the analytical expressions above, let us implement the numerical study of various types of phase transitions of Bose - Einstein condensate in ultra - cold Bose gases.

At the first, we deal with the symmetry restoration of condensate. To this end, in every case of interest the model parameters $\mu, \lambda$ and $\mathrm{m}$ are chosen to be close to those of experimental setting. To begin with, let us focus on what was observed in the first experimental realization of BEC: the appearance of BEC at given values of $\lambda$ and $\mathrm{m}$. Selecting, for example, $\lambda=10^{-11} \mathrm{eV}^{-2}, m=8.10^{10} \mathrm{eV}$ we consider the T dependence of condensate $\phi_{0}$ at several values of chemical potential. It is plotted in Fig. 1 which indicates that the critical temperature $T_{c}$ depends on $\mu$ as given in Eq. (31). On the other hand, Fig. 1 also reveals that the symmetry $\mathrm{U}(1)$, which was broken at $T=0$, gets restored at higher value of $T=T_{c}$. Fig.2, which displays the evolution of effective potential $\mathrm{V}$ against $\phi_{0}$ at several values of chemical potential $\mu$, proves that the restoration phase transition is second order.

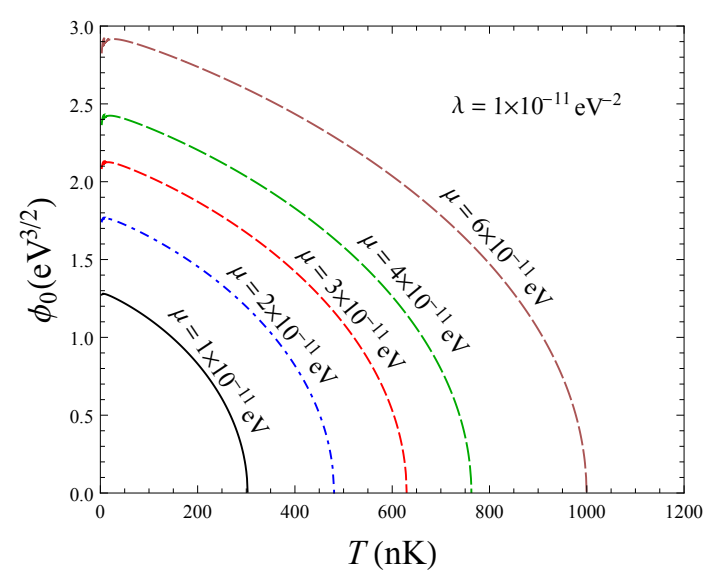

Fig. 1. The $\mathrm{T}$ dependence of order parameter $\phi_{0}$ at $\lambda=10^{-11} \mathrm{eV}^{-2}$ and several values of $\mu$

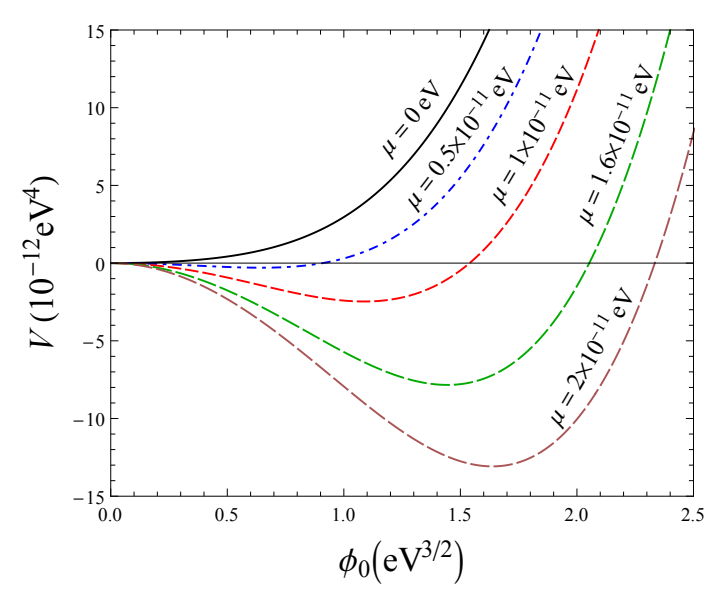

Fig. 2. The evolution of effective potential $\mathrm{V}$ against $\phi_{0}$ at $\lambda=10^{-11} \mathrm{eV}^{-2}$ and several values of $\mu$

Next, let us deal with another type of phase transition which is generated by changing the coupling constant $\lambda$ at fixed $\mu$.In Fig.3 we plot the T dependence of $\phi_{0}$ at several values of coupling constant $\lambda$ for $\mathrm{m}$ given above and $\mu=10^{-11} \mathrm{eV}$. It represents another scenario of Bose - Einstein condensation: the appearance of Bose -Einstein condensate depends on the coupling constant. Fig. 3 also confirms the restoration of $\mathrm{U}(1)$ as $\mathrm{T}$ increases from zero and the restoration transition is second order, as is shown in Fig. 4.

The phase diagrams of these restorations in the $(T, \mu)$ and $(T, \lambda)$ planes are presented in Figs.5 and 6, respectively. Their common feature is that there are two distinct regions of the model parameters: the BEC takes place only when $\bar{\mu}>0$, at $\bar{\mu}=0$ there occurs the restoration of broken symmetry. In addition, the behavior of phase diagram in Fig.6 is in accordance with Eq. (32). 


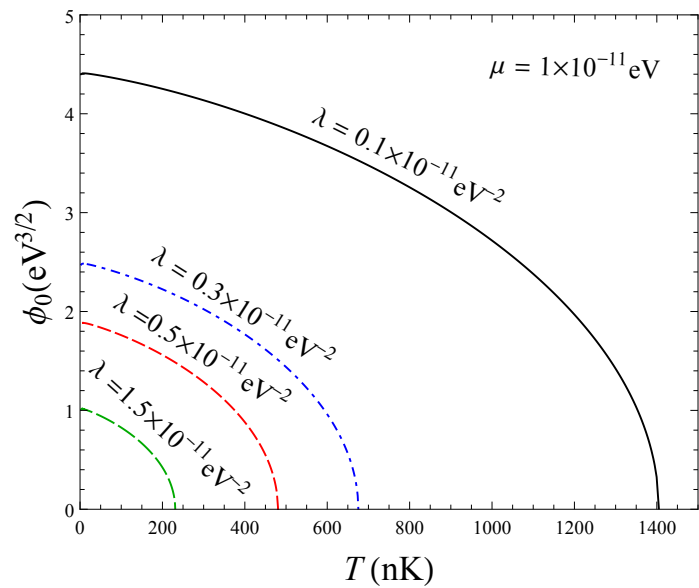

Fig. 3. The $\mathrm{T}$ dependence of order parameter $\phi_{0}$ at $\mu=10^{-11} \mathrm{eV}$ and several values of $\lambda$

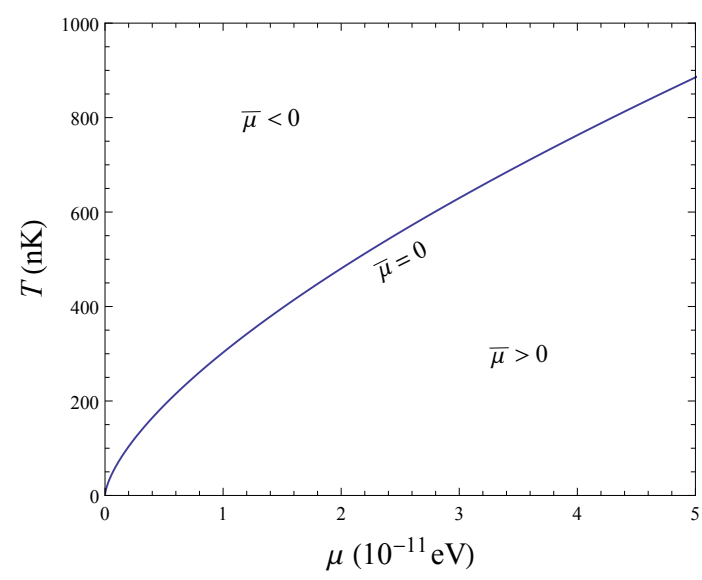

Fig. 5. Phase diagram of restoration transition in the $(T, \mu)$ - plane. The broken phase $(\bar{\mu}>0)$ and unbroken phase $(\bar{\mu}<0)$ are separated by the phase diagram $(\bar{\mu}=0)$

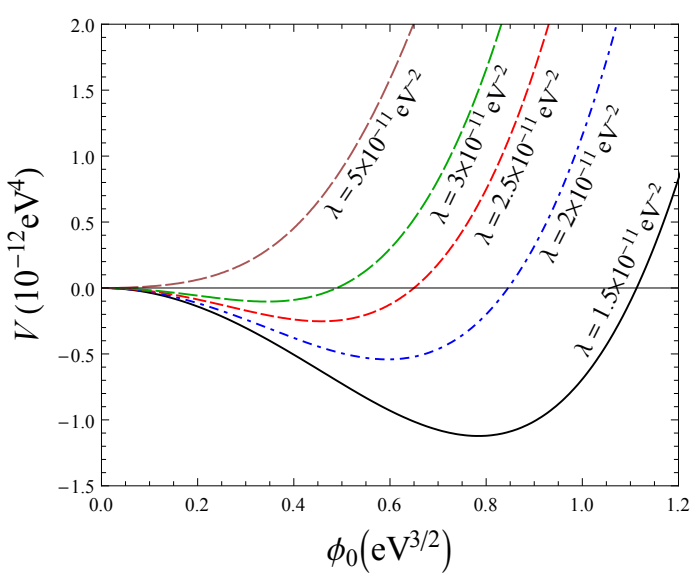

Fig. 4. The evolution of effective potential $\mathrm{V}$ against $\phi_{0}$ at $\mu=10^{-11} \mathrm{eV}$ and several values of $\lambda$

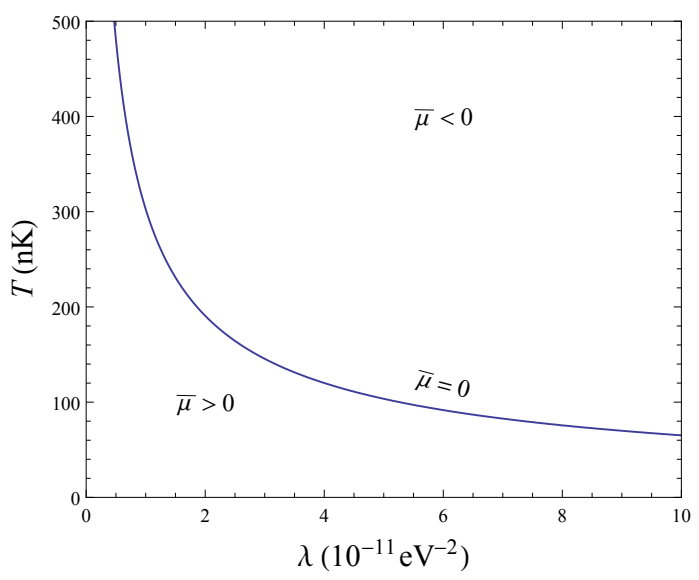

Fig. 6. Phase diagram of restoration transition in the $(T, \lambda)$ - plane. The broken phase $(\bar{\mu}>0)$ and unbroken phase $(\bar{\mu}<0)$ are separated by the phase diagram $(\bar{\mu}=0)$

\section{CONCLUSIONS}

In the preceding sections several physical properties of Bose - Einstein condensate were studied by means of the CJT effective potential in the improved double - bubble approximation which preserves the Goldstone theorem. The main results we found are in order:

1. It proved that the condensate is a superfluid in broken phase.

2. Several equations of state were established in the regimes of high and low temperature. 
3. The numerical investigation deals with two types of phase transition characterized by adjusting three parameters $\mathrm{T}, \mu$ and $\lambda$.

Here we discovered two difference types of phase structure of one - component Bose Einstein condensate: the conventional scenario of BEC manifests at given $\lambda$; the un - conventional scenario of BEC exhibits when $\lambda$ is made changed. The last phenomenon could be directly verified in Bose gas experiments at ultra low temperature.

\section{ACKNOWLEDGMENTS}

This research is funded by Vietnam National Foundation or Sciences and Technology Development (NAFOSTED) under grant FWO.103.2013.09.

\section{REFERENCES}

[1] M. H. Anderson, J. R. Ensher, M. R. Matthews, C. E. Wieman and E. A. Cornell, Science 269 (1995) 198.

[2] K. B. Davis, M. -O. Mewes, M. R. Andrew, N. J. Van Druten, D. S. Durfee, D. M. Kurn and W. Ketterle, Phys. Rev. Lett. 75 (1995) 3969.

[3] C. J. Myatt, E. A. Burt, R. W. Ghrist, E. A. Cornell and C. E. Wieman, Phys. Rev. Lett. 78 (1997) 586.

[4] D. S. Hall, M. R. Matthews, J. R. Ensher, C. E. Wieman and E. A. Cornell, Phys. Rev. Lett. 81 (1998) 1539.

[5] D. M. Stamper -Kurn, H. -J. Miesner, A. P. Chikkatur, S. Inouye, J. Stenger and W. Ketterle, Phys. Rev. Lett. 83 (1999) 661.

[6] G. Modugno, M. G. Modugno, F. Riboli, G. Roati and M. Inguscio, Phys. Rev. Lett. 89 (2002) 190404.

[7] H. -J. Miesner, D. M. Stamper - Korn, J. Stenger, S. Inouye, A. P. Chikkatur and W. Ketterle, Phys. Rev. Lett. 82 (1999) 2228.

[8] S. B. Papp, C. E. Wieman, Phys. Rev. Lett. 97 (2006) 180404.

[9] G. Thalhammer, G. Barontini, L. De Sario, J. Catani, F. Minardi and M. Inguscio, arXiv/ 0803.2763.

[10] E. Timmermans, Phys. Rev. Lett. 81 (1998) 5718.

[11] P. Ao and S. T. Chui, Phys. Rev. A 58 (1998) 4836.

[12] B. Van Schaeybroeck, Phys. Rev. A 78 (2008) 023624.

[13] T. -L. Ho and V. B. Shenoy, Phys. Rev. Lett. 77 (1996) 3276.

[14] I. E. Mazets, Phys. Rev. A 65, 033618 (2002).

[15] A. A. Svidzinsky and S. T. Chui, Phys. Rev. Lett. A 68 (2003) 013612.

[16] R. A. Barankov, Phys. Rev. A 66 (2002) 013612.

[17] J. O. Indekeu and B. Van Schaeybroeck, Phys. Rev. Lett. 210402 (2004) 93.

[18] Tran Huu Phat, Le Viet Hoa, Nguyen Tuan Anh and Nguyen Van Long, Ann. Phys. (NY) 324 (2009) 2074.

[19] K. Sasaki, N. Suzuki and H. Saito, Phys. Rev. A 83 (2011) 053606.

[20] T. Kadokura, T. Aioi, K. Sasaki, T. Kishimoto and H. Saito, Phys. Rev. A 85 (2012) 013602.

[21] S. Hayashi, M. Tsubota and H. Takeuchi, Phys. Rev. A 87 (2013) 063628.

[22] J. M. Cornwall, R. Jackiw and E. Tomboulis, Phys. Rev. D 10 (1974) 2428.

[23] G. Amelino - Camelia and S. Y. Pi, Phys. Rev. D 47 (1993) 2356.

[24] Tran Huu Phat, Nguyen Van Long, Nguyen Tuan Anh and Le Viet Hoa, Phys. Rev. D 78(2008) 105016. 